\title{
Association between food patterns and difficulties in falling asleep among adolescents in Norway - a descriptive Young-Hunt3 study
}

\author{
Beate André ${ }^{1,2} \cdot$ Helena Canhão ${ }^{3,4} \cdot$ Geir A. Espnes ${ }^{1,2}$ - Ana Maria Ferreira Rodrigues ${ }^{3,4}$. Maria Joao Gregorio ${ }^{3}$. \\ Camilla Nguyen $^{1,2} \cdot$ Rute Sousa $^{3,4} \cdot$ Kjersti Grønning ${ }^{1,2}$
}

Received: 29 November 2019 / Accepted: 27 February 2020 / Published online: 24 March 2020

(C) The Author(s) 2020

\begin{abstract}
Aim Adolescents' sleep duration has decreased over the past century; this is mainly caused by problems with falling asleep. Short sleep duration, poor sleep quality, and later bedtimes, in addition to difficulties in falling asleep, are further associated with adolescents' increased food intake, poor diet quality, and excess body weight. Obesity has become a major health worldwide problem, with increasing prevalence among several age groups. In addition, higher body mass index is one of the most consistently reported correlates of short sleep duration. The aim of this study was to study if short sleep duration, connected with problems falling asleep, is associated with obesity and weight gain among Norwegian adolescents.

Subject and methods This is a cross-sectional study using data from the Young-HUNT3 cohort study, which comprises the adolescent part of HUNT3. Young-HUNT3 includes data on 8217 participants aged 13 to 20 years old. Cluster analysis were used to categorize patterns of similarities in food consumption. Bivariate analyses were conducted to explore associations between difficulties in falling asleep and unhealthy food patterns.

Results The analyses found statistically significant differences $(<0.001)$ between adolescents having an unhealthy food pattern, higher BMIs, and more difficulties with falling asleep than adolescents with a healthy food pattern.

Conclusion This study shows that an unhealthy food pattern is associated with shorter sleep duration among Norwegian adolescents. Health promotion educational interventions focusing on disseminating knowledge to parents and adolescents about unhealthy food intake and other risk factors related to insufficient sleep ought to be developed.
\end{abstract}

Keywords Short sleep duration $\cdot$ Food patterns $\cdot$ Falling asleep $\cdot$ Adolescents

\section{Background}

A good night's sleep is critical for people's health, and is an important part in the natural process of restoration (Jerath et al.

Beate André

beate.andre@ntnu.no

Helena Canhão

helenacanhao@gmail.com

Geir A. Espnes

geirae@ntnu.no

Ana Maria Ferreira Rodrigues

anamfrodrigues@gmail.com

Maria Joao Gregorio

mariajoaobg@gmail.com

Camilla Nguyen

camilla.nguyen@ntnu.no
2014). There is accumulating evidence that shorter sleep duration is becoming a pervasive problem in today's society (Hasler et al. 2004). Reduction of sleep duration may results in metabolic alterations, including glucose intolerance (Spiegel et al.

Rute Sousa

rutedesousa@gmail.com

Kjersti Grønning

kjersti.gronning@ntnu.no

1 Department of Public Health and Nursing, NTNU Center for Health Promotion Research, Norwegian University of Science and Technology (NTNU), 7004 Trondheim, Norway

2 NTNU Center for Health Promotion Research, Trondheim, Norway

3 CEDOC, NOVA Medical School, University Nova de Lisbon, Lisbon, Portugal

4 Portuguese Society of Rheumatology, Lisbon, Portugal 
1999), confirming that sleep problems, especially among adolescents, need attention. Studies show that reduced sleep is associated with increased food intake among young males (Brondel et al. 2010). Furthermore, some adolescents also tend to have interest in more evening-type activities (Carskadon et al. 1993), challenging their "biological clock". Staying awake in the evenings, especially on weekend nights, makes it even harder to fall asleep on school nights (Owens and Group 2014).

The sleep duration of adolescents has decreased, but the decreased sleep duration also increases with higher age (Matricciani and Olds 2012). The National Sleep Foundation has assessed that adolescents need $9 \mathrm{~h}$ of sleep. Less than $8 \mathrm{~h}$ of sleep on school nights is considered an inadequate sleep length (Gradisar et al. 2013). Several studies have shown that inadequate sleep duration $(<8 \mathrm{~h} /$ day $)$ is related to numerous harmful health outcomes such as hypertension, diabetes, and risk of suicide in adults (Ayas et al. 2003; Cohen et al. 2009; Fitzgerald et al. 2011; Knutson and Van Cauter 2008; Kripke et al. 2002). Other studies of children and adolescents have shown associations between short sleep duration and low meal frequency (Stroebele et al. 2013), high intake of snacks and soft drinks (McKnight-Eily et al. 2011), and low intake of fruits and vegetables (Garaulet et al. 2011). A study of Norwegian adolescents has shown that sleep duration was associated with several health-risk behaviors, such as current smoking or snuffing, irregular meal patterns and intake of sweets, and poor academic achievement (Stea et al. 2014). Short sleep duration is also found to be associated with obesity in children and young adults (Nielsen et al. 2011).

Obesity has become a widespread, worldwide health problem, contributing to a major negative impact on human health (Lind et al. 2016). The massive increase in obesity observed over the previous 3 decades could involve a numerous of reasons, but it is mostly related to an increase in food consumption and a decrease in physical activity (Ogden et al. 2014). Dietary patterns characterized by high meat consumption are found to be associated with unhealthy life styles and depression symptoms in adults (Gregório et al. 2017). Unhealthy eating behaviors among adolescents has also been related to stressful situations such as low levels of social support and inadequate coping skill (Martyn-Nemeth et al. 2009).

Based on the presented background, the aim of this study is to investigate associations between BMI, unhealthy diets, and difficulties in falling asleep among adolescents, using epidemiological data from the Norwegian Young-HUNT3 study.

\section{Methods}

The HUNT Study in Norway includes a unique database of self-reported and clinical data on personal and family medical histories connected to health and lifestyle (Krokstad et al.
2013). The Young-HUNT3 population contained 8217 teenagers from the age of 13 to 20 years old. We have used descriptive statistics to explore the associations.

\section{Data collection}

In the Young-HUNT surveys, students in junior high and high school first received written information about the purpose of the study. For all junior high school students, a written consent from a parent or guardian was also required. This consent was distributed along with the written information, which was returned and signed at the school before being passed on to the HUNT Research Center. The students signed a written consent to participate in school before they were included in the study. Students who were absent on the day of the selfreported questionnaire session had to sign their consent when they participated in the clinical part of the survey. Youths absent from school got both the questionnaires and written consent by post to their home address; these were completed and returned by post to the HUNT Research Center.

\section{Demographic cohort}

Nord-Trøndelag County is situated in the middle of Norway and has a population size of about 130.000 inhabitants. The county is mostly rural with no large cities, but several small towns. The county is representative of Norway with regard to geography, economy, industry, sources of income, age distribution, morbidity, and mortality. From 2017 Nord-Trøndelag County merged with Sør-Trøndelag County and became Trøndelag County.

\section{Measurements}

The data in this study consist of measurements assessing BMI, diet, and sleeping habits. Sleeping habits were assessed using questions created by the Norwegian Institute of Public Health and especially for Young-HUNT, "During the last month have you: had difficulty falling asleep in the evening?". The question is also used in other studies assessing sleep habits (Sivertsen et al. 2015). Eating habits were measured using seven items of the validated Eating Attitudes Test (EAT) (Garner and Garfinkel 1979), while food consumption was collected by asking how often the participants normally ate or drank different kinds of food and drinks (e.g., fruits, vegetables, boiled potatoes, oily fish, whole-grain bread, white and semi-grain bread, chocolate/candy, pasta, sausages, water, sugar-free and sugary soft drinks, whole milk, and juice).

\section{Statistical analyses}

Data were analyzed using IBM SPSS Statistics (version 22). We used descriptive statistics to describe food patterns and chi-square statistics to explore food group consumption 
Table 1 Attendance rates for the Young-HUNT3 Survey

\begin{tabular}{lllll}
\hline Surveys & $\begin{array}{l}\text { Number invited: } \\
n\end{array}$ & $\begin{array}{l}\text { Response to } \\
\text { questionnaire: } n(\%)\end{array}$ & $\begin{array}{l}\text { Response to clinical } \\
\text { data: } n(\%)\end{array}$ & $\begin{array}{l}\text { Response to questionnaire } \\
\text { and clinical data: } n(\%)\end{array}$ \\
\hline Junior high & 5614 & $4749(84.6)$ & $4801(85.5)$ & $4615(82.2)$ \\
High school & 4357 & $3336(76.6)$ & $3294(75.6)$ & $3017(69.2)$ \\
Not in school & 493 & $115(23.3)$ & $97(19.7)$ & $84(17.0)$ \\
TOTAL & 10,464 & $8200(78.4)$ & $8192(78.3)$ & $7716(73.7)$ \\
\hline
\end{tabular}

frequency variations (André et al. 2017). A $p$ value of $<0.05$ was set as the cut-off point for statistical significance, but the phi coefficient from the chi-square statistics was also checked to evaluate the strength of the associations. Food patterns were identified with the use of cluster analysis (André et al. 2017). $\mathrm{K}$-means cluster analysis is appropriate for data with big sample dimensions (André et al. 2017). This analysis generates clusters with comparable characteristics (Gregório et al. 2017) where the respondents are categorized into distinct clusters (André et al. 2017). Food variables were re-coded into continuous variables by calculating the normal intake for 1 week (André et al. 2017). We then checked for and excluded extremes or outliers. Therefore, the continuous variables were consistent before consecutive rounds of K-means cluster analysis were used to confirm that the clusters were not predisposed by food groups with exact occurrence (Magidson and Vermunt 2002). The final model met the assumptions of K-means clustering.

\section{Ethical issues}

The information pamphlets for the Young-HUNT3 study were developed in cooperation with the Data Inspectorate of
Norway, the Health Directorate of Norway, and the Regional Committees for Medical and Health Research Ethics. The written consents from both the participants and their guardians were stored in the HUNT databank. Both HUNT and the Regional Committees for Medical and Health Research Ethics (REK, 2015/1211) have accepted this study.

\section{Results}

Table 1 presents the attendance rates for the Young-HUNT3 Survey, showing an attendance rate for both questionnaire and clinical data of $73.7 \%(n=7716)$.

Table 2 presents the descriptive data of BMI and how often the adolescents found it difficult to fall asleep in the evening.

\section{Food consumption}

More than half of the adolescents in the survey reported eating potato chips (53.2\%) and candy/chocolate (64.1\%) every week. Only approximately one third ate whole-grain bread several times a day, and almost half of the sample (47.3\%) consumed fish (salmon, trout, and mackerel) less than every week. Daily, a
Fig. 1 Cluster centers describe the average value for each variable within each cluster and reflect the characteristics of the typical case for each cluster

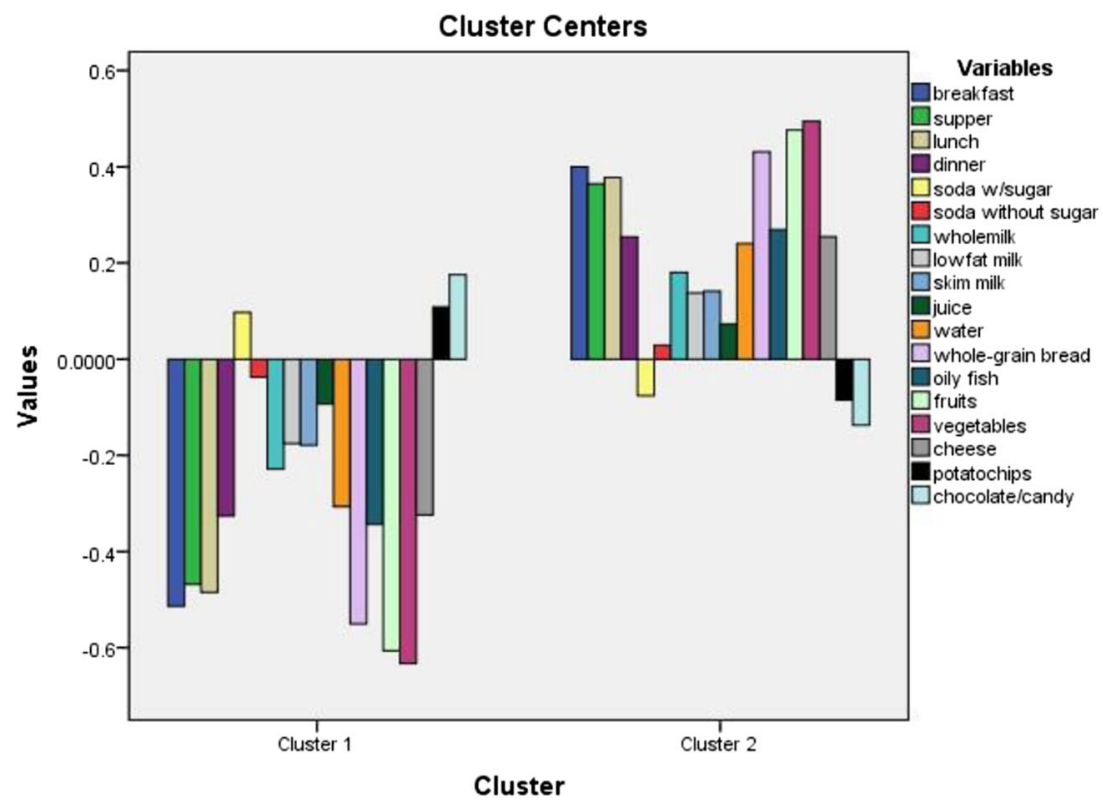


Table 2 Difficulty falling asleep and BMI, according to gender

\begin{tabular}{lccl}
\hline & Total: $n$ & Girls: $n(\%)$ & Boys: $n(\%)$ \\
\hline $\begin{array}{l}\text { How often have you during the last month had difficulty falling asleep in } \\
\text { the evening? }\end{array}$ & & & \\
Almost every night & 440 & $290 /(65.9)$ & $150 /(34.1)$ \\
Often & 810 & $513 /(63.3)$ & $297 /(36.7)$ \\
Sometimes & 3379 & $1867 /(55.3)$ & $1512 /(44.7)$ \\
Never & 3222 & $1328 /(41.2)$ & $1894 /(58.8)$ \\
TOTAL & 7851 & $3998 /(50.9)$ & $3853 /(49.1)$ \\
BMI & & & \\
$<25.0$ & 6211 & $3117(41.1 \%)$ & $3094(40.8 \%)$ \\
$25.0-29.9$ & 1061 & $524(6.9 \%)$ & $537(7.1 \%)$ \\
$\geq 30.0$ & 320 & $150(2.0 \%)$ & $170(2.2 \%)$ \\
\hline
\end{tabular}

significantly greater proportion of boys than girls consumed oily fish, potato chips, candy, chocolate, and cheese. A higher number of girls than boys consumed fruit and vegetables daily.

\section{Cluster analysis}

The cluster analysis resulted in two clusters based on similarities in reported food consumption among the adolescents. Cluster 1 consisted of adolescents with an unhealthy food pattern reporting a larger consumption of chocolate/candy, pasta, sausages, sugar-free and sugary soft drinks, whole milk, juice, and white and semi-grain breads than adolescents in cluster 2 , which had a healthy food pattern involving higher consumption of fruits, vegetables, boiled potatoes, oily fish, whole-grain bread, and water.

\section{Lifestyle characteristics}

Table 3 presents the results from the bi-variate analyses exploring differences between sleeping habits and food patterns (healthy cluster 2 versus unhealthy cluster 1), and between
BMI and the food patterns. These analyses show statistically significant associations between difficulties in falling asleep and adolescents with an unhealthy food pattern (cluster 1), and statistically significant associations between BMI and food patterns. There are fewer adolescents with an unhealthy food pattern (cluster 1) and low BMI, and there are more adolescents with an unhealthy food pattern (cluster 1) and high BMI.

\section{Discussion}

The aim of this study was to investigate the associations between BMI, food patterns, and difficulties in falling asleep among Norwegian adolescents. Our study confirmed findings from other studies (Gregório et al. 2017; Lien et al. 2001) showing that higher BMI is associated with eating unhealthy food. There may be, however, many reasons why adolescents eat unhealthily. Other studies have shown that poor eating habits also are linked to larger food intake, reduction in exercise (Lind et al. 2016), feeling stressed (Martyn-Nemeth et al. 2009), too much TV viewing, and inactivity (Pearson et al. 2014). However, other studies have found that adolescents who are living alone with little or no peer support have an unhealthier diet (Fitzgerald et al. 2013).

Findings in this study confirmed the findings from another study (Brondel et al. 2010), showing statistically significant associations between problems with falling asleep among adolescents and unhealthy food patterns. Other studies have shown associations between short sleep duration, low meal frequency (Stroebele et al. 2013), and high intake of snacks and soft drinks (McKnight-Eily et al. 2011), both food choices that are characteristics of an unhealthy diet.

Our bi-variate analyses detected that adolescents having difficulties with falling asleep also had an unhealthy food pattern and a higher BMI. To target adolescents' food intake, prevention programs designed to support health dietary choices are warranted (Fitzgerald et al. 2013). Furthermore,
Table 3 Difficulties with falling asleep, BMI, clusters 1 and 2

\begin{tabular}{lllll}
\hline & Total: $n(\%)$ & Cluster 1: $n(\%)$ & Cluster 2: $n(\%)$ & $P$ value \\
\hline Frequency of difficulty falling asleep in the evening during the last month & & $<0.001$ \\
Almost every night & $439(5.6 \%)$ & $272(7.9 \%)$ & $167(3.8 \%)$ & \\
Often & $808(10.3 \%)$ & $427(12.5 \%)$ & $381(8.6 \%)$ & \\
Sometimes & $3371(43.0 \%)$ & $1499(43.4 \%)$ & $1883(42.7 \%)$ & \\
Never & $3218(41.4 \%)$ & $1240(36.2 \%)$ & $1978(44.9 \%)$ & \\
TOTAL & $7836(100 \%)$ & $3427(100 \%)$ & $4409(100 \%)$ & \\
BMI & & & & \\
$<25$ & $6152(81.9 \%)$ & $2578(79.3 \%)$ & $3574(83.8 \%)$ & \\
$25-29.99$ & $1048(13.9 \%)$ & $504(15.5 \%)$ & $544(12.8 \%)$ & \\
$\geq 30$ & $315(4.2 \%)$ & $169(5.2 \%)$ & $146(3.4 \%)$ & \\
TOTAL & $7515(100 \%)$ & $3251(100 \%)$ & $4264(100 \%)$ & \\
\hline
\end{tabular}

Chi-square analyses, $p$ value $<0.05$ 
short sleep durations due to difficulties falling asleep are both a health and an educational advancement risk for adolescents (Ayas et al. 2003; Fitzgerald et al. 2011; Knutson and Van Cauter 2008; Kripke et al. 2002; Stea et al. 2014).

Previously, when giving adolescents lifestyle recommendations, the focus has been on food consumption and exercise; here, we show the importance of good sleep patterns, and point to the importance of including factors such as sleep quality and duration in such recommendations (Chaput 2014). Adolescents' overall health may be at risk by not getting an adequate amount of sleep. More attention towards innovative solutions to reduce poor sleeping habits are therefore needed (Chaput 2014). Health personnel, e.g., pediatricians (Owens and Group 2014) or school health nurses, have the potential to advocate for educational, administrative, and health policies that promote healthy sleep habits in adolescents. Health education classes and school-based interventions may also be valuable strategies to prevent unhealthy eating and to advocate better sleep patterns. To register patterns and changes among adolescents in relation to diet and food consumption, it will be important to collect longitudinal data that detects these changes (Gonsalves and Hawk, 2014).

\section{Strengths and limitations}

The strength of this study is that we are working with a population that has been found to be representative for the Norwegian population, with a detailed registration of food intake, BMI, and sleep patterns, making it possible to compare the findings from this study with other studies. Food patterns and diet are influenced by culture, making the validity and reliability of the food intake questionnaires culturally conditioned. This may be a limitation when comparing our findings with other studies from non-western cultures. Another limitation is the descriptive design, as it does not allow for causal explanations of the associations between difficulties in falling asleep and unhealthy food patterns. Another important limitation to address is the sleep pattern questions that were developed especially for the Young-HUNT 3 study and are thus not validated. This must be taken into consideration when comparing the findings of this study with other studies.

\section{Conclusion}

This study found a statistically significant association between unhealthy food patterns and difficulties in falling asleep among Norwegian adolescents. The mechanisms explaining why this association is present needs further investigation. The importance of sleep in relation to obesity should be included in lifestyle recommendations for adolescents, as these conventionally have focused only on food consumption and exercise. Thus, the knowledge about associations between BMI, food patterns, and difficulties in falling asleep among Norwegian adolescents should be used in developing educational programs for both parents and adolescents to promote healthier sleeping and eating habits.

Acknowledgments The Nord-Trøndelag Health Study (The HUNT Study) is a collaboration between HUNT Research Centre, (Faculty of Medicine and Health Sciences, NTNU, Norwegian University of Science and Technology), Trøndelag County Council, Central Norway Regional Health Authority, and the Norwegian Institute of Public Health.

Funding Information Open Access funding provided by NTNU Norwegian University of Science and Technology (incl St. Olavs Hospital - Trondheim University Hospital). The EEA and Norway Grants. The European Economic Area (EEA) Grants and Norway Grants represent the contribution of Iceland, Liechtenstein and Norway to reducing economic and social disparities and to strengthening bilateral relations with $16 \mathrm{EU}$ countries in Central and Southern Europe and the Baltics. Promoting Food Security in Portugal (ProFooSe Study) PortugalNorway.

Availability of data and materials The datasets used and/or analyzed during the current study are available from the corresponding authors upon reasonable request.

\section{Compliance with ethical standards}

Ethical approval and consent to participate The Regional Committees for Medical and Health Research Ethics in Norway have assessed and approved the HUNT3 study and the use of the data in this article (REK 2015/1211). The manuscript contains neither individual persons' data in any form nor other forms of sensitive information. Individuals participating have received information about the study and done it voluntarily, having first given their consent to participate. Consent forms were available at the HUNT center and at every school.

\section{Competing interests No conflicts of interest.}

Consent for publication Not applicable.

Open Access This article is licensed under a Creative Commons Attribution 4.0 International License, which permits use, sharing, adaptation, distribution and reproduction in any medium or format, as long as you give appropriate credit to the original author(s) and the source, provide a link to the Creative Commons licence, and indicate if changes were made. The images or other third party material in this article are included in the article's Creative Commons licence, unless indicated otherwise in a credit line to the material. If material is not included in the article's Creative Commons licence and your intended use is not permitted by statutory regulation or exceeds the permitted use, you will need to obtain permission directly from the copyright holder. To view a copy of this licence, visit http://creativecommons.org/licenses/by/4.0/.

\section{References}

André $B$ et al (2017) Is there an association between food patterns and life satisfaction among Norway's inhabitants ages 65 years and older? Appetite 110:108-115 
Ayas NT, White DP, Manson JE, Stampfer MJ, Speizer FE, Malhotra A, Hu FB (2003) A prospective study of sleep duration and coronary heart disease in women. Arch Intern Med 163:205-209

Brondel L, Romer MA, Nougues PM, Touyarou P, Davenne D (2010) Acute partial sleep deprivation increases food intake in healthy men. Am J Clin Nutr 91:1550-1559

Carskadon MA, Vieira C, Acebo C (1993) Association between puberty and delayed phase preference. Sleep 16:258-262

Chaput J-P (2014) Sleep patterns, diet quality and energy balance. Physiol Behav 134:86-91

Cohen S, Doyle WJ, Alper CM, Janicki-Deverts D, Turner RB (2009) Sleep habits and susceptibility to the common cold. Arch Intern Med 169:62-67

Fitzgerald A, Heary C, Kelly C, Nixon E, Shevlin M (2013) Self-efficacy for healthy eating and peer support for unhealthy eating are associated with adolescents' food intake patterns. Appetite 63:48-58

Fitzgerald CT, Messias E, Buysse DJ (2011) Teen sleep and suicidality: results from the youth risk behavior surveys of 2007 and 2009. J Clin Sleep Med 7:351-356

Garaulet $\mathrm{M}$ et al (2011) Short sleep duration is associated with increased obesity markers in European adolescents: effect of physical activity and dietary habits. The HELENA study. Int J Obes 35:1308-1317

Garner DM, Garfinkel PE (1979) The eating attitudes test: an index of the symptoms of anorexia nervosa. Psychol Med 9:273-279

Gonsalves D, Hawk H (2014) Goodenow C, Unhealthy weight control behaviors and related risk factors in Massachusetts middle and high school students. Matern Child Health J 18:1803-1813

Gradisar M, Wolfson AR, Harvey AG, Hale L, Rosenberg R, Czeisler CA (2013) The sleep and technology use of Americans: findings from the National Sleep Foundation's 2011 Sleep in America poll. J Clin Sleep Med 9:1291-1299

Gregório MJ et al (2017) Dietary patterns characterized by high meat consumption are associated with other unhealthy life styles and depression symptoms. Front Nutr 4:25. https://doi.org/10.3389/fnut. 2017.00025

Hasler G et al (2004) The association between short sleep duration and obesity in young adults: a 13-year prospective study. Sleep 27:661666

Jerath R, Harden K, Crawford M, Barnes VA, Jensen M (2014) Role of cardiorespiratory synchronization and sleep physiology: effects on membrane potential in the restorative functions of sleep. Sleep Med 15:279-288. https://doi.org/10.1016/j.sleep.2013.10.017

Knutson KL, Van Cauter E (2008) Associations between sleep loss and increased risk of obesity and diabetes. Ann N Y Acad Sci 1129:287304

Kripke DF, Garfinkel L, Wingard DL, Klauber MR, Marler MR (2002) Mortality associated with sleep duration and insomnia. Arch Gen Psychiatry 59:131-136
Krokstad S et al (2013) Cohort profile: the HUNT study. Norway Int J Epidemiol 42:968-977

Lien N, Lytle LA, Klepp K-I (2001) Stability in consumption of fruit, vegetables, and sugary foods in a cohort from age 14 to age 21. Prev Med 33:217-226. https://doi.org/10.1006/pmed.2001.0874

Lind L et al (2016) Uppsala consensus statement on environmental contaminants and the global obesity epidemic. Environ Health Perspect 124:A81-A83. https://doi.org/10.1289/ehp.1511115

Magidson J, Vermunt J (2002) Latent class models for clustering: a comparison with K-means. Can J Mark Res 20:36-43

Martyn-Nemeth P, Penckofer S, Gulanick M, Velsor-Friedrich B, Bryant FB (2009) The relationships among self-esteem, stress, coping, eating behavior, and depressive mood in adolescents. Res Nurs Health 32:96-109

Matricciani L, Olds T, Petkov J (2012) In search of lost sleep: secular trends in the sleep time of school-aged children and adolescents. Sleep Med Rev 16:203-211

McKnight-Eily LR, Eaton DK, Lowry R, Croft JB, Presley-Cantrell L, Perry GS (2011) Relationships between hours of sleep and healthrisk behaviors in US adolescent students. Prev Med 53:271-273

Nielsen L, Danielsen K, Sørensen T (2011) Short sleep duration as a possible cause of obesity: critical analysis of the epidemiological evidence. Obes Rev 12:78-92

Ogden CL, Carroll MD, Flegal KM (2014) Prevalence of obesity in the United States. JAMA 312:189-190

Owens J, Group ASW (2014) Insufficient sleep in adolescents and young adults: an update on causes and consequences. Pediatrics 134:e921e932

Pearson N, Biddle SJ, Williams L, Worsley A, Crawford D, Ball K (2014) Adolescent television viewing and unhealthy snack food consumption: the mediating role of home availability of unhealthy snack foods. Public Health Nutr 17:317-323

Sivertsen B, Harvey AG, Pallesen S, Hysing M (2015) Mental health problems in adolescents with delayed sleep phase: results from a large population-based study in Norway. J Sleep Res 24:11-18

Spiegel K, Leproult R, Van Cauter E (1999) Impact of sleep debt on metabolic and endocrine function. Lancet 354:1435-1439

Stea T, Knutsen T, Torstveit M (2014) Association between short time in bed, health-risk behaviors and poor academic achievement among Norwegian adolescents. Sleep Med 15:666-671

Stroebele N, McNally J, Plog A, Siegfried S, Hill JO (2013) The association of self-reported sleep, weight status, and academic performance in fifth-grade students. J Sch Health 83:77-84

Publisher's note Springer Nature remains neutral with regard to jurisdictional claims in published maps and institutional affiliations. 